\title{
The Effect of Goal Moderation on the Achievement and Satisfaction of Physical Activity Goals
}

DOI:

$10.1145 / 3432209$

\section{Document Version}

Accepted author manuscript

Link to publication record in Manchester Research Explorer

\section{Citation for published version (APA):}

Alqahtani, D., Jay, C., \& Vigo, M. (2020). The Effect of Goal Moderation on the Achievement and Satisfaction of Physical Activity Goals. ACM Proceedings on Interactive, Mobile, Wearable and Ubiquitous Technologies, 4(4), [116]. https://doi.org/10.1145/3432209

\section{Published in:}

ACM Proceedings on Interactive, Mobile, Wearable and Ubiquitous Technologies

\section{Citing this paper}

Please note that where the full-text provided on Manchester Research Explorer is the Author Accepted Manuscript or Proof version this may differ from the final Published version. If citing, it is advised that you check and use the publisher's definitive version.

\section{General rights}

Copyright and moral rights for the publications made accessible in the Research Explorer are retained by the authors and/or other copyright owners and it is a condition of accessing publications that users recognise and abide by the legal requirements associated with these rights.

\section{Takedown policy}

If you believe that this document breaches copyright please refer to the University of Manchester's Takedown Procedures [http://man.ac.uk/04Y6Bo] or contact uml.scholarlycommunications@manchester.ac.uk providing relevant details, so we can investigate your claim.

\section{OPEN ACCESS}




\title{
The Effect of Goal Moderation on the Achievement and Satisfaction of Physical Activity Goals
}

\author{
DEEMAH ALQAHTANI, The University of Manchester, United Kingdom \\ CAROLINE JAY, The University of Manchester, United Kingdom \\ MARKEL VIGO, The University of Manchester, United Kingdom
}

\begin{abstract}
Many self-trackers lose interest in, disengage from and ultimately withdraw from tracking. Reasons for this include poor motivation, unmet expectations and difficulty in attaining daily goals. To support users in reflecting on their goals more realistically, we developed FitReflect, an app that moderates physical activity goals by factoring in users' confidence in achieving the goals. The app also encourages users to reflect on their goals regularly and think about the factors affecting their achievement. We conducted a 4-week field experiment where we trialled the app with fourteen Fitbit users. We found that, compared with a non-moderated goal condition, participants with moderated goals achieved their goals more often, got closer to them, and adjusted them more frequently. Crucially, they were also more satisfied with their physical activity. More frequent goal updates were key to align user goals with their confidence and capabilities in achieving them.
\end{abstract}

CCS Concepts: • Human-centered computing $\rightarrow$ Empirical studies in HCI.

Additional Key Words and Phrases: self-tracking, wearable devices, physical activity, reflection, goal setting, self-efficacy, healthy adults

ACM Reference Format:

Deemah Alqahtani, Caroline Jay, and Markel Vigo. 2020. The Effect of Goal Moderation on the Achievement and Satisfaction of Physical Activity Goals. Proc. ACM Interact. Mob. Wearable Ubiquitous Technol. 4, 4, Article 0 ( 2020), 18 pages. https: //doi.org/10.1145/1122445.1122456

\section{INTRODUCTION}

With the help of wearable devices, self-trackers (i.e. people who track their own activity) monitor a wide range of variables including physical activity, productivity and sleep patterns. Typically, self-trackers expect tracking systems to provide them with insights that help them achieve the goals they set themselves. These insights can provide information about whether current behaviour should be maintained or adjusted in order to attain a given goal $[32,45]$ in what is termed the 'reflection stage' in personal informatics and self-tracking activity frameworks [31,32]. An insightful reflection, typically, facilitates behaviour change and/or maintenance of a positive behaviour.

However, self-trackers find it difficult to sustain tracking and continued reflection on their daily data if achieving their everyday goals proves infeasible [29]. Sudden or unexpected circumstances occurring during the day may affect goal achievement, which confound people's expectations [20]. Goal attainment may also be affected by

Authors' addresses: Deemah Alqahtani, The University of Manchester, Manchester, United Kingdom, deemah.alqahtani@manchester.ac.uk; Caroline Jay, The University of Manchester, Manchester, United Kingdom, caroline.jay@manchester.ac.uk; Markel Vigo, The University of Manchester, Manchester, United Kingdom, markel.vigo@manchester.ac.uk.

Permission to make digital or hard copies of all or part of this work for personal or classroom use is granted without fee provided that copies are not made or distributed for profit or commercial advantage and that copies bear this notice and the full citation on the first page. Copyrights for components of this work owned by others than ACM must be honored. Abstracting with credit is permitted. To copy otherwise, or republish, to post on servers or to redistribute to lists, requires prior specific permission and/or a fee. Request permissions from permissions@acm.org.

(C) 2020 Association for Computing Machinery.

2474-9567/2020/0-ART0 \$15.00

https://doi.org/10.1145/1122445.1122456

Proc. ACM Interact. Mob. Wearable Ubiquitous Technol., Vol. 4, No. 4, Article 0. Publication date: 2020. 
prior expectations, which influence motivation and performance [47]. In line with this, social cognitive theory (SCT) states that the perceived self-efficacy of individuals affects their subsequent behaviours and the goals they set to themselves [5]. Self-efficacy is defined as a person's self-perceived capability to perform a certain behaviour or attain a specific goal [6]. People with high self-efficacy tend to set higher goals and are more strongly motivated to achieve them [7]. Similarly, positive expectations are an incentive to perform well, while negative ones disincentivise performance [4].

There is evidence indicating that unmet expectations decrease satisfaction in many domains including careers [54] and healthcare [25]. Physical activity tracking is not exempt from this phenomenon in that inconsistencies between self-trackers' expectations about their data and what the data actually tells them causes frustration and disappointment $[1,26]$. Consequently, self-trackers might pause, suspend or even terminate their tracking $[13,18,24]$.

Based on the evidence that expectations moderate one's performance, our overarching hypothesis is that including expectations and beliefs about the users' capabilities in the self-tracking process will support them in achieving their goals. We also argue that moderating self-trackers' goals based on their perceived self-efficacy will help self-trackers engage with their actual goals and update them more frequently so they meet their fluctuating routines. Addressing this hypothesis is particularly relevant to everyday tracking practices such as tracking physical activity, as it may reduce the negative effect of unmet expectations, aid people in reflecting on their data and goals more realistically, update them more often and, finally, increase their motivation to achieve their goals.

In this paper, we attempt to bridge the gap between expectations and reality in physical activity tracking by explicitly enabling reflection on users' capabilities to achieve their goals while considering the contextual factors that affect their performance. To do so, we examine whether moderating physical activity goals based on the reported self-efficacy can help bridge this gap, and decrease the discomfort of unmet expectations. Specifically, we formulate the following hypotheses:

H1: Moderating physical activity goals based on daily perceptions of self-efficacy will increase goal achievement rate and closeness to goal.

H2: Moderating physical activity goals based on daily perceptions of self-efficacy will lead to users adjusting their goals more frequently.

H3: Moderating physical activity goals based on daily perceptions of self-efficacy will increase satisfaction about physical activity.

To test the above hypotheses, 14 self-trackers explored their physical activity data and reflected on their physical activity goals in a 4-week experiment. Participants reported the contextual, personal and social factors that affected their physical activity, and recorded their satisfaction with their performance, on a daily and weekly basis. Post-study questionnaires were used to collect participants' experiences with moderated goals, and the acquired insights. The contributions of this paper are:

- We use self-efficacy as a proxy for moderating self-trackers' physical activity goals.

- We develop FitReflect, an app that moderates self-trackers' daily goals using self-efficacy.

- We find that when goals are moderated, participants get closer to their primary goals, and are more satisfied with their physical activity.

- We find that when goals are moderated, users update their primary goals more frequently to align better their confidence and capabilities in achieving goals with their physical activity goals.

\section{RELATED WORK}

First, we outline the work conducted on physical activity tracking, engagement and reflection. Then, we examine works concerned with goal-setting strategies and types of goals. Finally, we explore the use of self-efficacy as a construct in self-tracking. 


\subsection{Self-tracking Physical Activity: Barriers to Engagement}

Physical activity is defined as any bodily movement that leads to energy expenditure [56]. Among the different types of data people track, physical activity is one of the most common. Existing literature has reported ways of measuring physical activity in different types of population [43, 53, 56], examined motivating the public to engage in physical activity courses [41] and explored methods and techniques for visualising physical activity data $[16,19,51]$. In personal informatics, most of the current tracking tools and wearables support physical activity and fitness tracking. Nonetheless, the high dropout rate when using these tools has prompted various investigations into the reasons for withdrawal [13, 29, 30]. Poor accuracy, lack of aesthetic appeal and lack of support for social tracking have all been shown to be detrimental to engagement with self-tracking [24]. Epstein et al. interviewed people whose activity tracking had lapsed, and suggested that activity tracking tools should be designed to take into account personal prior experiences such that data could be aggregated according to the characteristics and motivations of individuals [17]. Tang et al. studied self-trackers' engagement from the perspective of their adherence to long-term physical activity tracking [52]. They found that when people visually examined their tracking and goal adherence over a period of time, recognising the incompleteness of the their data gave them important insights. This led them to identify new wearing and activity patterns that would help them better utilise reflection over long-term physical activity data.

There are personal, psychological and physiological barriers that affect physical activity [46]. Current literature considers reflection as an integral part of tracking, and an indispensable opportunity to make sense of the data, assess the barriers that prevent goal achievement, and take actions accordingly [3, 9, 23, 31, 32]. Since it is known that a person's self-perceived capability to perform (i.e. self-efficacy) is a key determinant of physical activity [34], we argue there is an opportunity to incorporate self-efficacy in self-trackers' everyday reflection.

\subsection{Goal-Setting Strategies}

As people have variable physical activity routines, physical activity goals may need to respond to this fluctuation. Beyond the default recommendation of 10,000 step goal a day, research in personal informatics is increasingly supporting the notion that dynamic, evolving goals that are modified in response to contextual changes are better than those that remain static [21,37]. Consolvo et al. implemented different goal-setting approaches such as self-set goals, assigned goals and guided goals which motivated users to be physically active [14]. They found that most people preferred to self-set their goals or be guided by a fitness expert to know their most suitable goals. Munson and Consolvo have explored implementing a two-goal strategy by encouraging users to set primary and secondary goals [36]. The strategy works in two ways: first, it encourages users to achieve a second realistic goal if the first one becomes unattainable. Second, it pushes those who overachieved their first goal to achieve more with the secondary goal. Users reported that they found this strategy to be beneficial and motivating. Gouveia et al. found that the daily physical activity level of those who updated their goals when they were prompted to do so, is higher than those who stuck to their preset goals [21]. They suggested that goal-setting should be blended in self-tracking systems by allowing setting multiple concurrent goals that consider the changing levels of self-efficacy of individuals. Inspired by this, our work enables users explicitly state their daily self-efficacy in achieving their goals and then having their goals moderated as a result. We argue that by showing another form of daily goal achievement, one that reflects their perceived self-efficacy, self-trackers will engage with their actual goals and they will update them more frequently so they meet their fluctuating routines.

\subsection{Self-Efficacy in Personal Informatics}

Mobile health and behaviour change interventions are typically grounded in health behaviour theories [40,44]. Among these theories, social cognitive theory (SCT) has provided a conceptual base for health, promoting and monitoring interventions such as personalised health feedback [42], weight management [39] and nutrition [2]. 


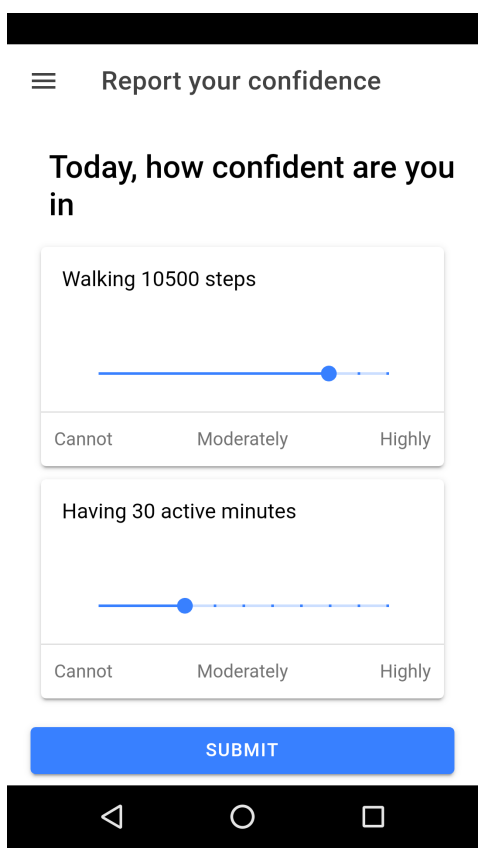

(a) Daily confidence

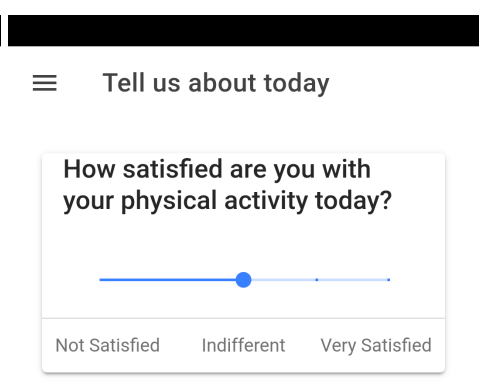

What affected your physical activity today?

$\checkmark$ Tiredness

$\square$ Pressure from work

$\checkmark$ Weather

$\square$ Personal problems

$\checkmark$ Physical discomfort

$\square$ Too much work

$\square$ Other interesting things to do

$\triangleleft$
O

(b) Daily reflection

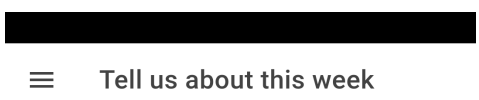

How satisfied are you with your physical activity this week?

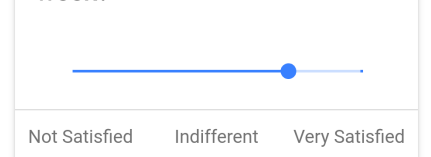

What action are you planning to take as a consequence? UPDATE MY GOAL NONE, I'LL STICK TO MY CURRENT GOAL

Fig. 1. Reporting workflow

A primary construct in SCT is self-efficacy, which describes a person's perceived belief or confidence in their ability to perform a specific action or achieve a certain goal [6]. Interestingly, this belief significantly influences the outcomes in that a person with high self-efficacy is likely to put high efforts towards reaching a goal and vice versa [50]. Choe et al. gave participants a hypothetical scenario of tracking a 10,000 step goal to examine how the framing of the received feedback influenced their perceived capabilities towards achieving the step goal. They found that, when the feedback highlighted the progress made (i.e. positive frame), individuals' perceived self-efficacy in achieving the goal was higher [12]. This study showed a promising role of self-efficacy in physical activity tracking in that individuals' beliefs about their capabilities of performing a task can be altered depending on the presented feedback. However, since the findings were based on a hypothetical scenario, we do not know whether they can be generalised to more ecologically valid settings.

To the authors' knowledge there are no works that incorporate self-efficacy in daily self-tracking. In this work, we enable self-efficacy to be explicitly reported each day and having goals moderated based on the reported values. Our work also encourages daily reflection on the contextual, personal and social factors that affect daily physical activity.

\section{THE FITREFLECT APP}

Inspired by the potential of self-efficacy as a construct to support the achievement of personal goals, we developed FitReflect, an Android mobile app to track physical activity goals and progress while logging daily self-efficacy and reflection. On set up, users have to connect the app with their Fitbit trackers and enter their age, gender, height, weight and their current or desired physical activity goal. We use steps and active minutes as measures of physical activity. Step count provides an indicator of movement, and active minutes is a measure of moderate to 
intense physical activity ${ }^{1}$ (i.e. brisk walking or cardio). A sensible physical activity goal would be expected to consider both of these measures, which are well-known data types in Fitbit trackers.

Figure 1 includes the screens involved in the reporting workflow. Figure 1a shows how, prompted by a daily morning push notification, users report their confidence in achieving their daily step and active minute goals. Confidence is reported through a slider that represents Bandura's self-efficacy scale [8] for measuring the strength of one's beliefs about achieving a goal. The original scale is a 100-point scale with 10-unit intervals from 0 to 100 . We used the simplified format of the scale which retains its structure but uses single unit intervals ranging from ' 0 ' for 'Cannot do' through ' 5 ' for 'Moderately certain can do' to ' 10 ' for 'Highly certain can do' [8]. Once users submit their confidence in the morning, they can check their progress towards the goals by switching to the daily progress screen on Figure 2b. The daily progress screen consists of two daily ring charts showing their progress towards their primary goals and two daily ring charts showing their progress towards that day's moderated goals. The moderated goal is computed based on the user's self-efficacy score (i.e. reported morning confidence) and their primary goal such that the moderated goal $(M)$ is the primary goal $(G)$ multiplied by the self-efficacy score $(S E)$ and divided by 10 representing the units of Bandura's self-efficacy scale [8] as indicated in the following formula:

$$
M=\frac{G \times S E}{10}
$$

The literature suggests that some users do not update their goals downwards because they perceive it to be a failure, which leads to systematic underachievement of their goals [49]. FitReflect bypasses this problem as users are not requested to update their goals on a daily basis but they have their goals moderated by FitReflect (although this moderation is indirectly caused by their self-efficacy reports). Therefore the moderated goal becomes a

${ }^{1}$ Active minutes are computed by Fitbit although the specific details of the algorithm are not available. See https://help.fitbit.com/articles/en_ US/Help_article/1379

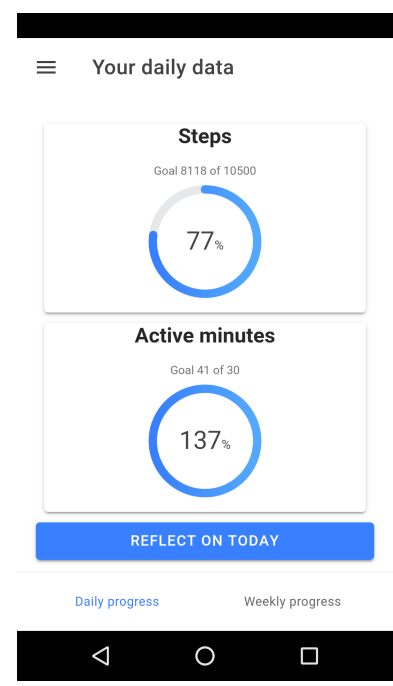

(a) Daily progress in the nonmoderated condition

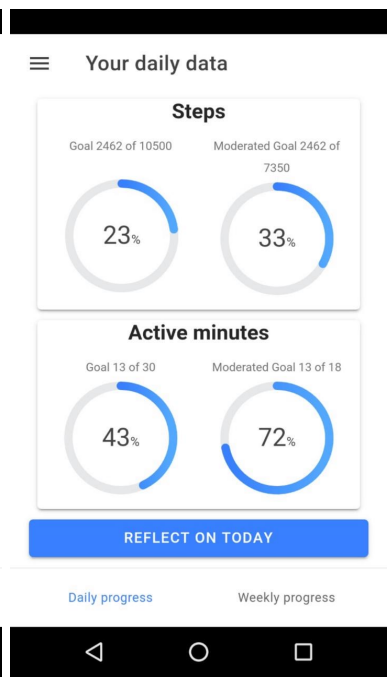

(b) Daily progress in the moderated condition

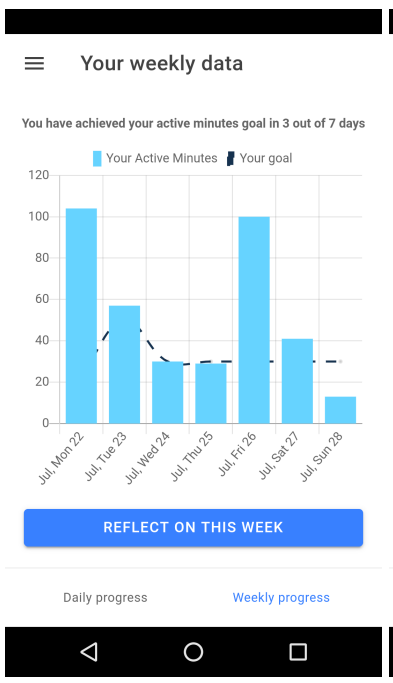

-(c) Weekly progress in the nonmoderated condition

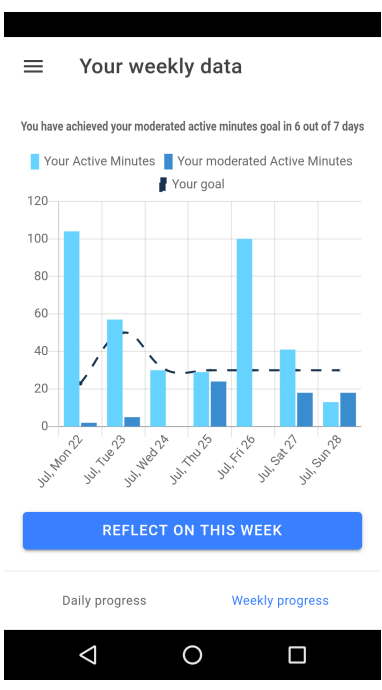

(d) Weekly progress in the moderated condition

Fig. 2. Tracking screens: non-moderated condition (Figures 2a and 2c) and moderated condition (Figures 2b and 2d) 
secondary goal in addition to the primary goal, which can still be updated anytime or when triggered by weekly reflection.

Even if participants can check their progress towards the goals at any time, FitReflect sends a push notification in the evening to remind participants to check their progress and submit their daily reflection. Then, in the screen depicted by Figure 1b, users rate their satisfaction with their physical activity on a 5-point slider. They can also select the factors that affected their physical activity that day by ticking on a set of checkboxes that were derived from the contextual, personal and social factors reported by Bandura as possible barriers to regular exercise routines including weather, tiredness and pressure from work [6]. Users also have the option to add further factors that are not included in the default checkbox list.

Weekly progress visualisations depicted in Figure $2 \mathrm{~d}$ enable users to compare their performance against their primary goals and the moderated goals in the last week. Twice a week, participants are reminded to reflect on their week hitherto (see Figure 1c) which involves, again, checking their weekly progress first and reporting their satisfaction on their weekly progress on a 5-point slider. Users are also asked whether they want to update their current goals or stick to them and, optionally, report any actions they intend to take. The rationale for this is that, typically, most self-tracking tools encourage users to set their goals at the point of first use. Yet, even if users can update their goals, most of them decide not to [49]. Sjöklint et al. suggest that by recommending concrete goals, self-tracking tools may impose an anchoring effect on the users, which is a cognitive bias exhibited when there is a reliance on the first piece of information encountered (the anchor), affecting the judgements that follow [55]. In this case, users get anchored by their goals and although some might establish their own baseline after a period of tracking, most users will stick to their status-quo (i.e. not doing anything about it). We argue that including an explicit goal adjusting functionality in the reporting workflows will help self-trackers reflect on their goals more effectively. In this way, they can decide if more realistic goals should be set informed by their current progress and perceived self-efficacy.

\section{METHOD}

The goal of the experiment was to test the impact of incorporating daily self-efficacy reporting and subsequent moderation of physical activity goals on self-trackers' progress and satisfaction with their physical activity. We also examined whether daily reflection on contextual, social and personal barriers to physical activity helped self-trackers better understand what hindered and helped their physical activity routines.

\subsection{Participants}

We recruited 14 self-trackers who (1) described themselves as lightly to moderately physically active but may not achieve their goals every day (2) owned and used a Fitbit activity tracker for at least one month. These inclusion criteria were enforced in a screening interview. Participants were recruited through snowball sampling, social media posts and word-of-mouth. Ten of the participants were female and four male, and they were on average 26.43 years old $(M d n=25.5, S D=4.14)$. After completion, each participant received a $£ 20$ voucher as an incentive for taking part. Table 1 shows participant demographic information as well as their activity level, satisfaction with their physical activity, Fitbit model and the length of time they had been self-tracking.

\subsection{Data Collection}

The study had three main stages: an online entry survey; a four week within-subjects field experiment; and an online exit questionnaire. The purpose of the entry survey was to collect demographic information, self-reported physical activity levels (light, moderate or vigorous) and their current satisfaction with their ability to achieve physical activity goals on a 5-point Likert scale. Table 1 on columns 5 and 6 shows the initial satisfaction and physical activity level for each participant. Nine of the participants considered themselves moderately active, 
Table 1. Participants' demographic information (leftmost four columns), activity level, satisfaction with their physical activity, Fitbit model and the length of time they had been self-tracking

\begin{tabular}{|c|c|c|c|c|c|c|c|}
\hline ID & Gender & Age & Occupation & Physical activity level & How satisfied & Fitbit model & Tracking period \\
\hline P01 & M & 26 & Content developer & Moderate & Neutral & Charge 3 & $5 \mathrm{mos}$ \\
\hline $\mathrm{P} 02$ & $\mathrm{~F}$ & 21 & Student & Light & Neutral & Inspire & $4 \mathrm{mos}$ \\
\hline P03 & M & 25 & Fresh graduate & Moderate & Dissatisfied & Charge HR & $2 \mathrm{yr}$ \\
\hline P04 & $\mathrm{F}$ & 24 & Assistant psychologist & Moderate & Very dissatisfied & Charge 2 & $2 \mathrm{yr}$ \\
\hline P05 & $\mathrm{F}$ & 26 & Fashion design consultant & Moderate & Satisfied & Alta & $2 \mathrm{yr}$ \\
\hline P06 & $\mathrm{F}$ & 24 & Fresh graduate & Moderate & Dissatisfied & Charge 2 & $3 \mathrm{yr}$ \\
\hline P07 & $\mathrm{F}$ & 33 & Graduate student & Moderate & Satisfied & Charge 2 & $1 \mathrm{yr}, 10 \mathrm{mos}$ \\
\hline P08 & $\mathrm{F}$ & 22 & Secretary & Light & Dissatisfied & Alta & $3 \mathrm{yr}$ \\
\hline P09 & M & 22 & Student & Moderate & Satisfied & Alta & $2 \mathrm{yr}$ \\
\hline P10 & M & 33 & Shift charge engineer & Moderate & Dissatisfied & Versa & $5 \mathrm{mos}$ \\
\hline P11 & $\mathrm{F}$ & 27 & Graduate student & Light & Dissatisfied & Charge 2 & $1 \mathrm{yr}, 8 \mathrm{mos}$ \\
\hline $\mathrm{P} 12$ & $\mathrm{~F}$ & 29 & Graduate student & Light & Dissatisfied & Charge 2 & $1 \mathrm{mo}$ \\
\hline $\mathrm{P} 13$ & $\mathrm{~F}$ & 24 & Graduate student & Moderate & Satisfied & Charge 3 & $1 \mathrm{mo}$ \\
\hline P14 & $\mathrm{F}$ & 34 & Project manager & Light & Neutral & Charge 3 & $2 \mathrm{yr}$ \\
\hline
\end{tabular}

while five were lightly active. Four participants were satisfied with their daily goal achievement, three were neutral, six were dissatisfied and one participant was very dissatisfied.

In order to assess the effects of moderation on goal achievement, we also developed a baseline version of the app that did not display the moderated goals. The baseline condition involves the same reporting workflow as in Figure 1 although the progress tracking screens do not show the moderated goals in the charts, just their progress towards their primary goals - see Figures 2a and 2c respectively. Participants were split equally into two conditions, the baseline condition and the moderated condition. Figure 3 illustrates the screens and tasks involved in each of the conditions. The experimental design was within-subjects (where the gender distribution was the same between groups) such that participants in Group A started with the moderated goal condition, whereas Group B started with the baseline condition. After two weeks they switched conditions. We chose a within-subjects design so that participants could discuss goal moderation relative to a baseline condition in the exit survey.

Before starting, participants were sent a tutorial explaining the features of FitReflect and the tasks they were expected to do. Each participant received a specific version of the tutorial depending on which condition they were in. The tutorial in the moderated condition described the relationship between the reported confidence in achieving the goals and the moderated goal. Once the field experiment was completed, participants filled in an online exit questionnaire including open-ended questions about what they liked and disliked about having their goals moderated, and the insights they acquired during the experiment.

\subsection{Data Analysis}

Our analysis was divided into two phases on completion of the field experiment. The first was a quantitative phase to test the hypothesises we formulated. We evaluated the effect of moderation on the following dependent variables:

- Goal achievement rate. This variable represents the ratio of the number of days the goal was achieved divided by the total number of days. A higher value indicates a greater number of goals achieved. Goal changes were considered such that the daily physical activity was compared to the latest primary goal the participant had set. 


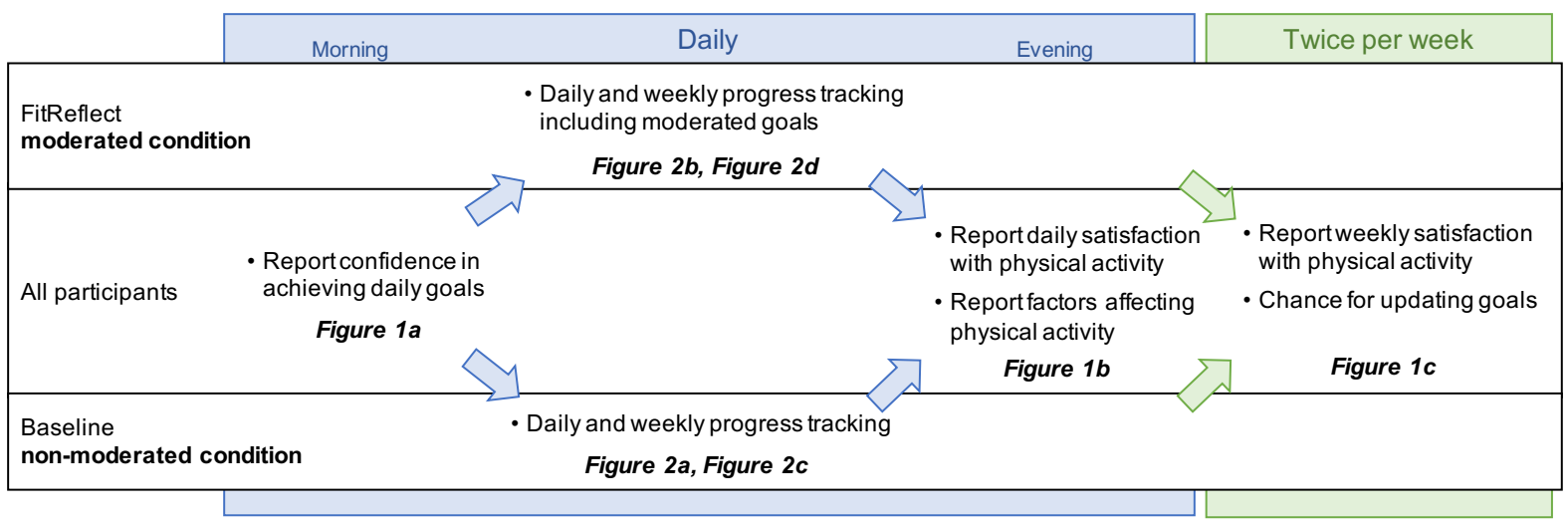

Fig. 3. Experiment workflows per condition

- Closeness to goal. This variable represents how close participants got to their actual physical activity goals by computing average daily physical activity divided by the goal of the day: $\frac{1}{N} \sum_{i=1}^{N} \frac{\text { physical activit }_{i}}{\text { goal }_{i}}$, where $N$ $=$ number of active days. The higher the value, the closer the participant got to the primary goal. Obviously, the goal can be surpassed so Closeness to goal can be $>1$. Goal changes were considered such that the daily physical activity value was divided by the latest primary goal the participant had set.

- Goal change. The number of times participants adjusted their primary goals as a result of the reflection that took place twice a week.

- Satisfaction. Daily and weekly satisfaction with their physical activity on 5-point scale where '1' was 'Very dissatisfied' and '5' was 'Very satisfied'.

We computed Closeness to goal and Goal achievement rate for the two physical activities tracked: steps and active minutes. In a second qualitative analysis phase, we conducted thematic analysis on the responses received in the exit questionnaire by familiarising ourselves with the data, recording initial ideas, generating preliminary codes to contextualise the data, and iteratively reviewing the themes [11]. We used NVivo 12 for Mac to support the analysis of the data. To test the reliability of the coding, an independent researcher assessed the transcripts against the codebook.

\section{RESULTS}

We first describe the results of the field experiment, and then report the results of the thematic analysis of participants' responses to the exit questionnaire. We collected 748 self-efficacy reports ( $95 \%$ completion rate), 369 daily satisfaction responses (94\%), 112 for weekly satisfaction (100\%), and 408 for daily reflection, accounting for 1,229 responses.

We use parametric tests and the mean when the normality assumption is met as per the Shapiro-Wilk test, and non-parametric tests and the median when the normality assumption is not met. We use a Bonferroni adjustment to protect posthoc pairwise comparisons against Type I error. We also analyse if there were order effects by examining the interactions between the within-subjects factor (condition: moderated vs non-moderated) and the between-subjects factor (order: moderation first, Group A vs non-moderated baseline first, Group B), which we report when the interactions are significant. 


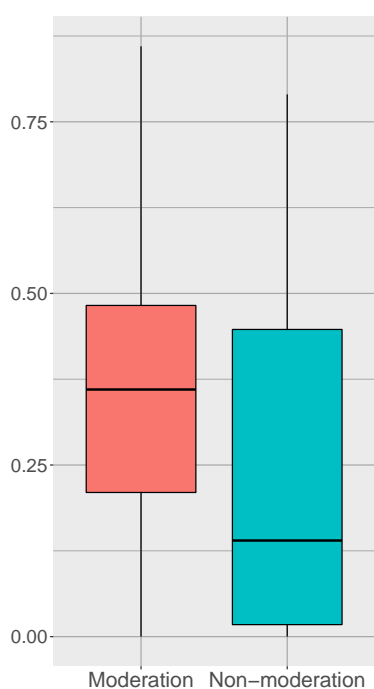

(a) Steps

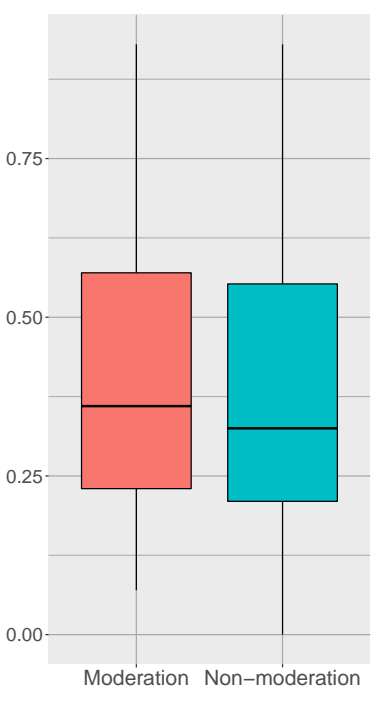

(b) Active minutes

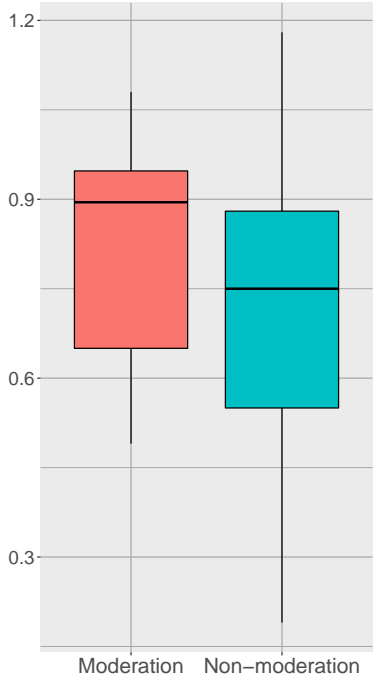

(a) Steps

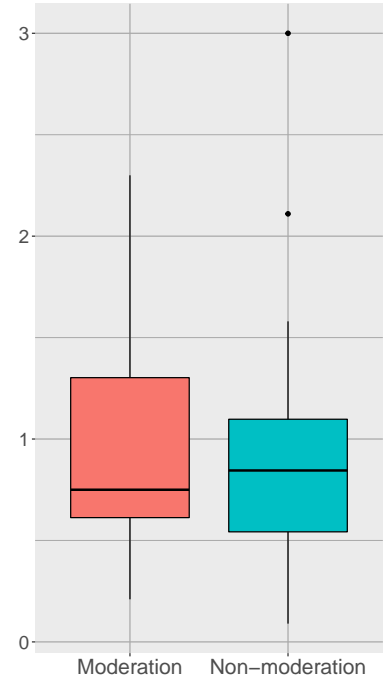

(b) Active minutes

Fig. 4. Goal achievement rate

Fig. 5. Closeness to goal

\subsection{Field Experiment}

An average of 108087 steps were taken in the moderated condition $(S D=28927)$ while 96921 steps we taken in the non-moderated one $(S D=37812)$. A two-way repeated measures ANOVA suggests there is an effect of moderation on the number of steps $(F(1,12)=5.64, p<0.05)$, and an interaction between condition and order, $F(1,12)=9.47, p<0.01$. Post-hoc pairwise comparisons indicate that the differences are significant for those in Group A, who took fewer steps after moderation was removed, $p<0.02$.

When it comes to active minutes, participants were more active in the moderated condition (497 active minutes, $S D=298)$ than in the non-moderated one (449 active minutes, $S D=286$ ). A two-way repeated measures ANOVA indicates there is no effect of moderation on active minutes although there is an interaction between condition and order, $F(1,12)=6.52, p<0.03$ which is not significant according to post-hoc pairwise comparisons $(p>0.05)$.

The median step goal was the same in both conditions $(M d n=10000)$ although the mean was lower in the moderated condition $(M=9142, S D=1511)$ than in the non-moderated one $(M=9642, S D=928)$. A Friedman test suggests there is no effect of moderation on the step goal set, $\chi^{2}(1)=1.8, p=0.18$. Analogously, active minutes goals are the same $(M d n=30)$ and, again, the mean is lower in the moderated condition $(M=36.79$, $S D=18.77)$ than in the non-moderated one $(M=38.57, S D=19.85)$ although there is no effect of moderation on active minutes goals, $\chi^{2}(1)=0.2, p=0.65$.

Although participants could not see the moderated goals in the baseline condition, moderated goals can still be calculated as participants reported their confidence anyhow: the moderated step goal was higher in the moderated condition $(M=6303, S D=1612)$ than in the non-moderated one $(M=5578, S D=1079)$. There is an effect of moderation on the moderated step goal according to a two-way repeated measures ANOVA $(F(1,12)=5.08, p<0.05)$ although pairwise comparisons suggest these differences are not significant. Similarly, the moderated goals for active time were higher in the moderated condition $(M=25.05, S D=15.16)$ than in the non-moderated one $(M=23.38, S D=15.56)$. This time there is no effect of moderation on moderated active minutes goals, $F(1,12)=3.36, p=0.09$. 
5.1.1 Goal achievement rate. As suggested by the boxplots in Figure 4a, the Goal achievement rate for steps was higher in the moderated condition $(M d n=0.36, S D=0.25)$ than in the non-moderated condition $(M d n=0.14$, $S D=0.29)$. A Friedman test indicates there is an effect of moderation on the goal achievement rate for daily steps, $\chi^{2}(1)=5.3, p<0.03$, which is significant according to a post-hoc Conover test $(p<0.001)$. For active minutes (see boxplots in Figure $4 \mathrm{~b}$ ), participants in the moderated condition $(M=0.43, S D=0.24)$ performed slightly better than the non-moderated condition $(M=0.39, S D=0.28)$. A two-way repeated measures ANOVA indicates there are no effects of moderation on the achievement rate of active minutes $(F(1,12)=0.77, p=0.40)$.

We compute the moderated goal achievement rate as well, which was very similar for steps: $M=0.67, S D=0.18$ for the moderated condition and $M=0.68, S D=0.21$ for the non-moderated one, $F(1,12)=0.01, p=0.93$. Results are similar for active minutes where it is higher for the moderated condition $(M=0.44, S D=0.25)$ than on the nonmoderated one $(M=0.39, S D=0.28)$ although the difference is again non significant, $F(1,12)=1.20, p=0.29$.

5.1.2 Closeness to goal. Closeness to goal for daily steps suggests that participants in the moderated condition got closer to their goal $(M=0.82, S D=0.20)$ than those the non-moderated condition $(M=0.72, S D=0.26)$ -see Figure 5a. A two-way repeated measures ANOVA suggests there is an effect of moderation on closeness to step goals, $F(1,12)=7.9, p<0.02$. Post-hoc pairwise comparisons confirm these effects are significant for the participants of Group A. Closeness to active minutes goal (see the boxplots in Figure 5b) was higher in the non-moderated condition $(M d n=0.85, S D=0.78)$ than in the moderated one $(M d n=0.75, S D=0.61)$ although a Friedman test indicates that moderation has no effect on it, $\chi^{2}(1)=0.08, p=0.78$.

5.1.3 Goal change. Participants in both conditions updated their steps and/or active minutes goals as a result of the reflection taking place twice a week. Five people changed their step goals in the moderated condition, whereas only two did it in the non-moderated condition. While the difference in the number of participants who updated their step goals is not significant $\left(\chi^{2}(1)=0.76, p=0.38\right)$, the participants in the moderated condition updated their goals nine times while those in the non-moderated condition did it only three times. Similarly, seven participants updated their active minutes goals in the moderated condition, while five did it on the non-moderated one $\left(\chi^{2}(1)=0.15, p=0.70\right)$, accounting for eleven updates in the moderated condition and seven in the non-moderated one.

5.1.4 Satisfaction. Self-reported satisfaction measures were taken on a daily basis, as part of the daily reflection. Participants in the moderated condition were more satisfied with their physical activity $(M d n=4, S D=1.37$, unl $)$ than those in the non-moderated condition $(M d n=3, S D=1.51$, liı). We computed the effect of moderation on daily satisfaction using condition and order as independent variables in a two-way ordinal regression. The results suggest that moderating the goals has a significant effect on daily satisfaction $\chi^{2}(1)=21.24, p<0.001$. Moreover, the results indicate there is an interaction between condition and order $\chi^{2}(1)=139.7, p<0.001$. Post-hoc pairwise comparisons confirm that the decrease in daily satisfaction from $M d n=4$ (m) to $M d n=3$ (n...) in Group $\mathrm{A}$ is significant when moderation is removed in the second period, $p<0.01$. Similarly, the later introduction of moderation in Group B increases satisfaction from $M d n=3$ (..1I) to $M d n=4$ (...11) and these participants are more satisfied than their Group A counterparts in the non-moderated condition $(p<0.001)$.

The satisfaction reports collected twice a week corroborate what daily satisfaction scores suggest: participants in the moderated condition $(M d n=4, S D=1.26$, .nin) were more satisfied than those in the non-moderated condition $(M d n=3, S D=1.34$, III!). We computed the effect of moderation on daily satisfaction using condition and order as independent variables in a two-way ordinal regression. We observe a similar pattern as in daily satisfaction whereby goal moderation has an effect on weekly satisfaction $\chi^{2}(1)=8.07, p<0.01$. Again, we find interactions between condition and order $\chi^{2}(1)=59.69, p<0.001$, although pairwise comparisons suggest differences are not significant $(p>0.05)$. 
Table 2. Summary of the results in the moderated condition: + indicates there is an effect of moderation on the differences, while $*$ indicates there is an effect and it is significant

\begin{tabular}{lll|l}
\hline & & Steps & Active minutes \\
\hline \multirow{3}{*}{ Activity and goals } & Amount/number & Higher* & Higher \\
& Moals & Lower & Marginally lower \\
& Goal achievement rate & Higher $^{*}$ & Marginally higher \\
& Closeness to goal & Higher* & Lower \\
Dependent variables & Goal change & Higher & Higher \\
& Daily Satisfaction & Higher* & Higher* \\
& Weekly satisfaction & Higher $^{+}$ & Higher $^{+}$ \\
\hline
\end{tabular}

Summary. Table 2 outlines the results in this section which indicates that participants' activity and moderated goals were in line: when participants were more active, their moderated goals were accordingly higher, whereas when they were less active, their moderated goals were lower. While it is known that self-efficacy is a predictor of performance [4], we confirm this may be the case for self-tracking of physical activity. Because of more frequent goal changes in the moderated condition, the main goals were adapted more frequently than in the non-moderated condition. Consequently, those in the moderated condition had goals that were more achievable, which led to a significantly higher goal achievement, closeness to goal and satisfaction. This is confirmed by Figure 6 where the area around the lines represents the interquartile range of the goals (top line) and steps (bottom line): in the moderated condition (Figure 6a), the overlap between the areas is larger than in non-moderated one (Figure 6b) because the goals and activity are more aligned to each other.

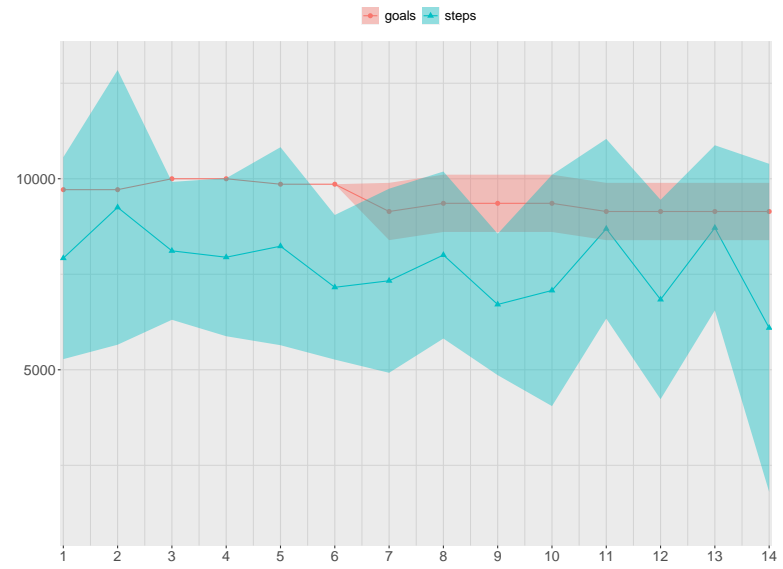

(a) Moderated

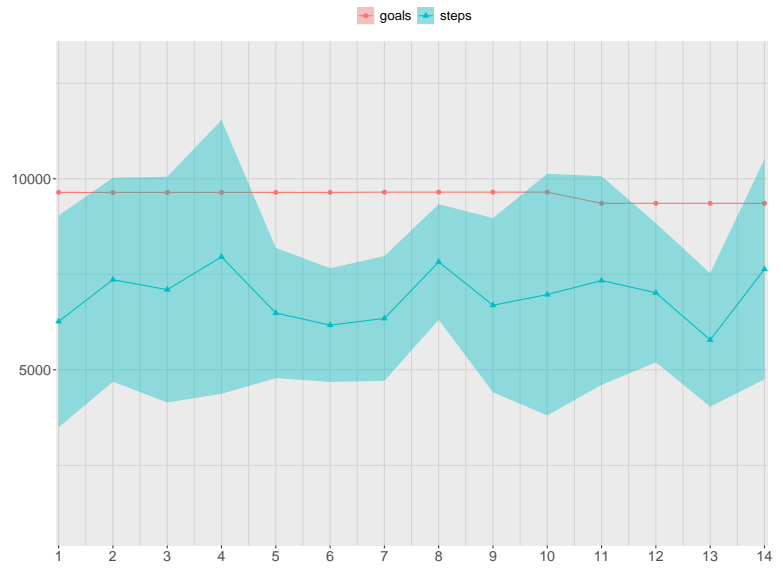

(b) Non-moderated

Fig. 6. Goals and steps over time: the line charts represent the means of the daily goal and steps while the surrounding area conveys the spread through the interquartile range 
Table 3. The top five barriers and facilitators affecting physical activity

\begin{tabular}{|c|c|c|c|c|c|c|c|}
\hline \multicolumn{4}{|c|}{ Non-moderated } & \multicolumn{4}{|c|}{ Moderated } \\
\hline Barriers & Frequency & Facilitators & Frequency & Barriers & Frequency & Facilitators & Frequency \\
\hline Tiredness & 38 & Shopping & 3 & $\begin{array}{l}\text { Personal prob- } \\
\text { lems }\end{array}$ & 20 & Shopping & 4 \\
\hline $\begin{array}{l}\text { Too much } \\
\text { work }\end{array}$ & 25 & $\begin{array}{l}\text { Trip and } \\
\text { walking }\end{array}$ & 3 & Tiredness & 20 & Travelling & 3 \\
\hline $\begin{array}{l}\text { Personal prob- } \\
\text { lems }\end{array}$ & 21 & Gym & 2 & $\begin{array}{l}\text { Too much } \\
\text { work }\end{array}$ & 15 & Football & 2 \\
\hline Weather & 16 & $\begin{array}{l}\text { Nice } \\
\text { weather }\end{array}$ & 1 & Weather & 14 & $\begin{array}{l}\text { Indoor walk- } \\
\text { ing }\end{array}$ & 2 \\
\hline $\begin{array}{l}\text { Other interest- } \\
\text { ing things to } \\
\text { do }\end{array}$ & 15 & $\begin{array}{l}\text { Playing in } \\
\text { the theme } \\
\text { park }\end{array}$ & 1 & $\begin{array}{l}\text { Other interest- } \\
\text { ing things to } \\
\text { do }\end{array}$ & 10 & $\begin{array}{l}\text { Nice } \\
\text { weather }\end{array}$ & 2 \\
\hline
\end{tabular}

5.1.5 Contextual, personal and social factors. On the daily reflection screen (see Figure 1b), participants reported the perceived contextual, personal and social barriers to achieve their physical activity goals. Factors that were not included in the default checkbox list were also reported, which included barriers as well as facilitators that enabled participants to achieve their goals or increased their satisfaction with their physical activity. Table 3 shows the top five most frequently reported barriers and facilitators affecting physical activity in each condition. We computed the Kendall rank correlation tests between the top ten reported barriers and facilitators in both conditions. The results show an almost a linear correlation: $\tau=0.73, p<0.05$ for the top-10 barriers and $\tau=0.78, p<0.05$ for the top-10 facilitators. This suggests that barriers and facilitators may have affected similarly to participants in both conditions.

\subsection{Qualitative Analysis}

Below we report the codes identified by the thematic analysis of the verbatim responses. The inter-rater reliability results showed a near-full agreement, Cohen's $\kappa=0.85$. We provide the number of participants that reported each theme in parentheses.

A taste of achievement (6). Participants appreciated the sense of accomplishment that moderated goals provided. As P02 described: "Experiencing the taste of achievement helps boosting your confidence leading to more enthusiasm." Participants indicated that most of the time they were aiming to achieve their moderated goal at the very least: "Having my goal moderated makes me always try to at least achieve it when I feel like I can't achieve the daily one due to sickness or work or any other reason, that did help me feel better about my daily activity and not regretting that I didn't achieve my goals that day" (P06).

Being realistic (7). Participants reported that moderating their goals helped them to think realistically about them. For example, P07 identified two benefits of goals' moderation: "Updating my confidence every day was good for me in two ways: being reasonable about what I could achieve for that day, and challenge myself to achieve more than my prediction which made me feel satisfied at night if I did it". This encouraged participants in forming a reflective practice on goals: "Setting a realistic goal and achieving it each day builds good habits and becomes part of your lifestyle" (P02).

Increased motivation, decreased disappointment (10). Participants reported that the moderation of goals kept them on track: “It helped me to be on track for my goal and not to lose hope, maybe today I didn't do it but 
tomorrow I will do it. Consistency and keeping me motivated are the main advantages" (P12). Having the primary and moderated goals available gave P05 a sense of accomplishment: "It motivates me to do my best and achieve my goals... whether I achieve my overall goal or the moderated one." Also, participants reported that the moderated goal helped reduce disappointment due to not achieving the primary goals. P04 acknowledged that expecting a "suitable goal" helped avoid disappointment, and P11 described how the moderated goal had her feel less guilty and more motivated: "When I see that I have completed the moderated goals in the graphs, it made me feel less guilty about not meeting the actual goal. Also, it gave me a sense of accomplishment and motivated me to work better in order to meet my actual goal ... After completing the experiment, I missed the application. It kept me motivated and I learned about my daily and weekly progress in a more informative form than on the Fitbit app."

Self-contract (3). Some participants reported that submitting daily confidence values increased their commitment to achieving what they set out to: "Every day when I submit my confidence, I consider it as a promise that has to be fulfilled" (P09). P03 described the daily confidence reporting as a 'self-contract': "Waking up and starting my day by telling how confident I am to achieve my goal was like a self-contract. I noticed that whenever I put high confidence scores, my mind keeps telling me 'You said you'll achieve your goal, don't forget!' and this motivates me so, by the end of the day, I achieve my goal or at least more than half of it."

Reflecting on barriers to goal achievement (4). Reflecting on their day and reporting what affected their physical activity led participants to identify unanticipated barriers, or barriers that they repeatedly encountered that prevented them from achieving their goals. P14 described the reflection process as "finding the reason to confront myself with it, if any obstacle happens." P11 said: "I knew there were activities that kept me from meeting my actual goal but reporting them explicitly in the application helped me find a solution. The most serious problem was a personal one, which gave me the insight that I need to work on managing my time better. It could sound obvious but reporting it consistently made it very clear to me."

\section{DISCUSSION}

We start this section by identifying the main findings and re-visiting our hypotheses. Then, we discuss the findings in relation to goal-setting and fostering reflection. Finally, we highlight avenues for future work.

\subsection{Findings}

Participants reported their self-efficacy to have their goals moderated accordingly. We found that incorporating self-efficacy in the daily reflection process can help self-trackers think about their goals more effectively. FitReflect helped participants to schedule reflection on a daily basis, forming a sustained reflective practice whereby participants kept monitoring and reflecting on their expectations, progress and the context affecting their physical activity. Being consistent about reflection boosted participants' motivation, engagement and achievements. Below, we revisit the three hypotheses we formulated at the outset:

H1. Moderating physical activity goals based on daily perceptions of self-efficacy will increase goal achievement rate and closeness to goal.

There was a positive effect of goal moderation on goal achievement rate and closeness to goal for step count. The moderated goals, which were based on the users' confidence and capabilities, mirrored daily physical activity in line with the social cognitive theory principles [50]. As reported by participants in Section 5.2, moderated goals were perceived to be more realistic and attainable goals without losing the sense of achievement. We acknowledge, as some participants reported, there may be a problem with moderated goals being set too low just so they can be achieved. However, our findings suggest that moderated goals were higher in the moderated condition, in accordance with the amount of activity. 
Some participants conveyed the idea that moderated goals became minimum achievable goals towards the primary goal, which echoes the secondary goals proposed by Munson and Consolvo [36]. Therefore, the primary goal could be perceived as an "ideal" goal, whereas a moderated goal can act as an stepping stone towards it. Hence, the moderated goal bridges the gap between what the users can actually achieve and what the users aspire to achieve. Having said this, we prefer not to be prescriptive about whether the moderated goal should be a backup goal, an alternate goal or a minimum goal. Instead, letting users appropriate a secondary goal may be a better strategy [15]. In this study, the moderated goal was a good enough goal for some, a trigger for reflection for many, and for others it was a stepping stone, a motivator to achieve the primary goal or a self-contract they felt committed to.

There was no effect of moderation on active minutes goal achievement. This could be because users cannot easily quantify active minutes in that one minute of physical activity does not always result in one active minute. Consequently, actions taken by users to achieve active minutes goals may not always be successful.

H2. Moderating physical activity goals based on daily perceptions of self-efficacy will lead to users adjusting their goals more frequently.

We observed more adjustments of the primary goal in the moderated condition. It is known that users do not update their goals downwards because they perceive it as a personal failure [49]. Delegating on FitReflect to have their goals moderated while still having a primary goal may have overcome this problem. It could also be that reflecting on one's capabilities (through the moderated goal) as well as the barriers and facilitators to goal achievement encouraged users in the moderate condition to update their goals more often, which is known to be an indicator of goal achievement [21]. Indeed, we observe that primary goals were lowered over time so that they were more aligned with user activity -see Figure 6. Yet, the difference in the number of participants who updated their step count goals at least once is not significant. This suggests that some participants iterated over their goals [38], while other participants' initial goal was already appropriate or needed minor adjustments.

H3. Moderating physical activity goals based on daily perceptions of self-efficacy will increase satisfaction about physical activity.

There was an effect of goal moderation on satisfaction. When participants could view moderated physical activity goals, they were more satisfied with their physical activity. This effect was found to be significant for both daily and weekly satisfaction. In line with goal-setting theory [33], this outcome may be related to $H 1$ in that users were better at achieving their primary goals and getting closer to them. Participants talked about how achieving moderated goals helped them "feel better" about their daily activities and "not regretting" that they did not achieve the primary goal. It also mitigated the guilt caused by underachievement of goals [49].

\subsection{Goal Moderation for Fostering Reflection}

The fluctuating nature of users' goals leads to the misalignment between their goals and the system goals [22]. It is also known that setbacks are common in behaviour change interventions due to the challenges faced in everyday life [27]. These are some of the main barriers to engagement with achievement-based goals such as those in physical activity tracking systems, which can be addressed by frequent reflection on goals. Personal informatics research has widely focused on the process of reflection in self-tracking. Breakdown, inquiry and transformation are the three conceptual dimensions for designing technologies with reflection in mind [9]. When it comes to physical activity tracking, reflection is also manifested under these dimensions [1]. In our experiment, through moderated goals, we attempted to foster a sustained reflective practice on physical activity goals. This practice encouraged users to think about their capabilities, their goals and the barriers in relation to their goals: participants reflected when they set their confidence in achieving their primary goals and when the goals were not achieved by thinking about the factors that influenced the lack of achievement. We found that encouraging a 
sustained reflective practice helped creating a sense of consistency in tracking. As with secondary goals [36], goal moderation has helped increasing people's motivation to do their best and feel satisfied about it, potentially reducing the chances of withdrawal. Below we describe the specific actions that sustained reflective practice and goal moderation have fostered.

6.2.1 Adjusting goals. Frequent reflection on physical activity goals helped the participants to think about their goals more objectively and more realistically. During this process, participants updated their goals to adjust their expectations according to their current circumstances. Our findings confirm the importance of blending goal-setting in daily self-tracking [21]. Our results, also, support the idea that providing an explicit functionality to reflect on goals more frequently can help alleviate the anchoring effect that default goals might introduce [49].

6.2.2 Enriched Reflection. While reflection took place in both conditions, participants in the moderated goal condition gained additional information from viewing their moderated goals. This translated into the frequent attempts of some participants to set more attainable goals. When users became aware of the gap between their expectations and reality they tailored their goals accordingly. Consequently they were more satisfied with their physical activity and more committed to achieving their goals.

6.2.3 Reflection on Context. By reporting and viewing the factors that affected their ability to attain their goals, participants were able to assess the factors that bridged or widened the gap between expectations and reality. This form of reflection can alter individuals' thinking patterns and induce behaviour change [10]. In this study, reporting contextual factors helped increase awareness of the barriers that occurred regularly, spot unexpected barriers, and identify facilitators to goal achievement.

\subsection{Limitations}

We acknowledge that the findings of our study are generalisable to our demographics and duration of the study. While we found that goal moderation held potential for our sample of users, it would be interesting to examine the effect with different age groups/conditions such as elderly, people with sedentary lifestyle or people with specific health conditions. Further, we do not discard the presence of Hawthorne effect [28], whereby individuals alter their behaviours when they know they are being observed, which might have influenced on how well the participants responded to our reminders.

Although a four week experiment has produced promising outcomes about the effect of goal moderation on goal achievement, we do not know whether this effect will hold in the long term. It is well known that attrition is a problem in long terms studies involving self-reporting on mobile devices [35] and the use of physical activity self-trackers [48]. Our findings suggest that goals and moderated goals tend towards convergence. In the long term these two goals may mirror each other entailing that users may be conditioned to set achievable goals as opposed to goals that challenge themselves to achieve more. It may also be the case that users learn to set goals that are achievable and challenging at the same time. These would be goals that require sacrifice and effort in order to be tackled but it would be attainable challenges at the same time. We pose that the potential benefits of tackling underachievement could encourage longer term use and counterbalance disengagement.

\subsection{Recommendations for Future Work}

In light of our findings, we suggest research avenues to leverage the relationship between expectations, progress and context in self-tracking.

6.4.1 Achievement-sensitive goal moderation. While we found that goal moderation increased goal achievement, low self-efficacy might have negatively affected such commitment. Some participants reported that setting a too low self-efficacy might have sometimes discouraged them from achieving their actual goals. For this reason, we 
propose that goal moderation could be calculated in a way that not only considers users' self-efficacy but their goal achievement history. Based on this calculation, the moderated goal could be set slightly higher when low self-efficacy values are submitted.

6.4.2 Tailoring self-efficacy reporting frequency. An individual's self-efficacy is idiosyncratic and can change unexpectedly. While some participants' kept their self-efficacy consistent for a few days, others changed it dramatically every day. One participant indicated that her confidence level might change in the middle of the day compared to what she submitted when the day started. This calls for enabling users to report self-efficacy as many times as they like, which would have implications on how the dependent variables are computed.

6.4.3 Modelling self-efficacy and context. A person's self-efficacy relies on her own beliefs, which could be supported or contrasted with the contextual factors. We propose modelling self-efficacy, context and physical activity progress so that users can explore the relationships between the three factors. For example, contextual factors that lead to high physical activity when low self-efficacy is reported have an actionable potential. Showing the relationship between these factors and progress can facilitate reflection and behaviour change. Functionalities such as those in personal informatics systems that utilise context (e.g. https://exist.io) could be leveraged to indicate the existence of such relationships.

\section{CONCLUSION}

In this paper, we examine the effect of moderating self-trackers' goals based on their perceived self-efficacy. We found that, when physical activity goals are moderated, self-trackers adjust their primary goals more often and, consequently, goal achievement is higher. As a result, self-trackers are more satisfied about their physical activity when their goals are moderated, as goal moderation leads them not only to higher achievement rates, but also to better understanding of their capabilities in achieving their goals, and the factors that hinder and facilitate tracking. They also report being more motivated and committed to achieving both their moderated and primary goals.

\section{ACKNOWLEDGEMENTS}

We thank all the participants who got involved in the study. We are also grateful for the reviewers' constructive feedback.

\section{REFERENCES}

[1] Deemah Alqahtani, Caroline Jay, and Markel Vigo. 2020. The Role of Uncertainty as a Facilitator to Reflection in Self-Tracking. In Proceedings of the 2020 ACM Designing Interactive Systems Conference (Eindhoven, Netherlands) (DIS '20). Association for Computing Machinery, New York, NY, USA, 1807-1818. https://doi.org/10.1145/3357236.3395448

[2] Eileen S Anderson, Richard A Winett, and Janet R Wojcik. 2007. Self-regulation, self-efficacy, outcome expectations, and social support: social cognitive theory and nutrition behavior. Annals of behavioral medicine 34, 3 (2007), 304-312.

[3] Amid Ayobi, Paul Marshall, and Anna L Cox. 2016. Reflections on 5 years of personal informatics: rising concerns and emerging directions. In Proceedings of the 2016 CHI Conference Extended Abstracts on Human Factors in Computing Systems. ACM, 2774-2781.

[4] Albert Bandura. 1986. Social foundations of thought and action. Englewood Cliffs, Nf 1986 (1986).

[5] Albert Bandura. 1991. Social cognitive theory of self-regulation. Organizational behavior and human decision processes 50, 2 (1991), $248-287$.

[6] Albert Bandura. 1997. Self-efficacy: The exercise of control. Macmillan.

[7] Albert Bandura. 2004. Health promotion by social cognitive means. Health education \& behavior 31, 2 (2004), 143-164.

[8] Albert Bandura. 2006. Guide for constructing self-efficacy scales. Self-efficacy beliefs of adolescents 5, 1 (2006), 307-337.

[9] Eric PS Baumer. 2015. Reflective informatics: conceptual dimensions for designing technologies of reflection. In Proceedings of the 33rd Annual ACM Conference on Human Factors in Computing Systems. ACM, 585-594. 
[10] Julie L Booth, Kelly M McGinn, Christina Barbieri, Kreshnik N Begolli, Briana Chang, Dana Miller-Cotto, Laura K Young, and Jodi L Davenport. 2017. Evidence for cognitive science principles that impact learning in mathematics. In Acquisition of complex arithmetic skills and higher-order mathematics concepts. Elsevier, 297-325.

[11] Virginia Braun and Victoria Clarke. 2006. Using thematic analysis in psychology. Qualitative research in psychology 3, 2 (2006), 77-101.

[12] Eun Kyoung Choe, Bongshin Lee, Sean Munson, Wanda Pratt, and Julie A Kientz. 2013. Persuasive performance feedback: The effect of framing on self-efficacy. In AMIA annual symposium proceedings, Vol. 2013. American Medical Informatics Association, 825.

[13] James Clawson, Jessica A Pater, Andrew D Miller, Elizabeth D Mynatt, and Lena Mamykina. 2015. No longer wearing: investigating the abandonment of personal health-tracking technologies on craigslist. In Proceedings of the 2015 ACM International foint Conference on Pervasive and Ubiquitous Computing. ACM, 647-658.

[14] Sunny Consolvo, Predrag Klasnja, David W McDonald, and James A Landay. 2009. Goal-setting considerations for persuasive technologies that encourage physical activity. In Proceedings of the 4th international Conference on Persuasive Technology. 1-8.

[15] Sunny Consolvo, Predrag Klasnja, David W. McDonald, and James A. Landay. 2014. Designing for Healthy Lifestyles: Design Considerations for Mobile Technologies to Encourage Consumer Health and Wellness. Found. Trends Hum.-Comput. Interact. 6, 3-4 (April 2014), 167-315. https://doi.org/10.1561/1100000040

[16] Daniel Epstein, Felicia Cordeiro, Elizabeth Bales, James Fogarty, and Sean Munson. 2014. Taming data complexity in lifelogs: exploring visual cuts of personal informatics data. In Proceedings of the 2014 conference on Designing interactive systems. ACM, 667-676.

[17] Daniel A Epstein, Jennifer H Kang, Laura R Pina, James Fogarty, and Sean A Munson. 2016. Reconsidering the device in the drawer: lapses as a design opportunity in personal informatics. In Proceedings of the 2016 ACM International foint Conference on Pervasive and Ubiquitous Computing. ACM, 829-840.

[18] Daniel A Epstein, An Ping, James Fogarty, and Sean A Munson. 2015. A lived informatics model of personal informatics. In Proceedings of the 2015 ACM International Joint Conference on Pervasive and Ubiquitous Computing. ACM, 731-742.

[19] Chloe Fan, Jodi Forlizzi, and Anind K Dey. 2012. A spark of activity: exploring informative art as visualization for physical activity. In Proceedings of the 2012 ACM Conference on Ubiquitous Computing. ACM, 81-84.

[20] Russell E Glasgow. 2008. Perceived barriers to self-management and preventive behaviors. Health behavior constructs: theory, measurement and research. Bethesda, MD: National Cancer Institute (2008).

[21] Rúben Gouveia, Evangelos Karapanos, and Marc Hassenzahl. 2015. How do we engage with activity trackers? A longitudinal study of Habito. In Proceedings of the 2015 ACM International foint Conference on Pervasive and Ubiquitous Computing. $1305-1316$.

[22] Rebecca Gulotta, Jodi Forlizzi, Rayoung Yang, and Mark Wah Newman. 2016. Fostering Engagement with Personal Informatics Systems. In Proceedings of the 2016 ACM Conference on Designing Interactive Systems (Brisbane, QLD, Australia) (DIS '16). Association for Computing Machinery, New York, NY, USA, 286-300. https://doi.org/10.1145/2901790.2901803

[23] Kirsi Halttu and Harri Oinas-Kukkonen. 2017. Persuading to reflect: role of reflection and insight in persuasive systems design for physical health. Human-Computer Interaction 32, 5-6 (2017), 381-412.

[24] Daniel Harrison, Paul Marshall, Nadia Bianchi-Berthouze, and Jon Bird. 2015. Activity tracking: barriers, workarounds and customisation. In Proceedings of the 2015 ACM International Joint Conference on Pervasive and Ubiquitous Computing. ACM, 617-621.

[25] Jeffrey L Jackson and Kurt Kroenke. 2001. The effect of unmet expectations among adults presenting with physical symptoms. Annals of internal medicine 134, 9_Part_2 (2001), 889-897.

[26] Da-jung Kim, Yeoreum Lee, Saeyoung Rho, and Youn-kyung Lim. 2016. Design opportunities in three stages of relationship development between users and self-tracking devices. In Proceedings of the 2016 CHI Conference on Human Factors in Computing Systems. ACM, 699-703.

[27] Predrag Klasnja, Sunny Consolvo, and Wanda Pratt. 2011. How to Evaluate Technologies for Health Behavior Change in HCI Research. In Proceedings of the SIGCHI Conference on Human Factors in Computing Systems (Vancouver, BC, Canada) (CHI'11). Association for Computing Machinery, New York, NY, USA, 3063-3072. https://doi.org/10.1145/1978942.1979396

[28] Henry A Landsberger. 1958. Hawthorne Revisited: Management and the Worker, Its Critics, and Developments in Human Relations in Industry. (1958).

[29] Amanda Lazar, Christian Koehler, Joshua Tanenbaum, and David H Nguyen. 2015. Why we use and abandon smart devices. In Proceedings of the 2015 ACM International Joint Conference on Pervasive and Ubiquitous Computing. ACM, 635-646.

[30] Hyunho Lee and Youngseok Lee. 2017. A look at wearable abandonment. In 2017 18th IEEE International Conference on Mobile Data Management (MDM). IEEE, 392-393.

[31] Ian Li, Anind Dey, and Jodi Forlizzi. 2010. A stage-based model of personal informatics systems. In Proceedings of the SIGCHI conference on human factors in computing systems. ACM, 557-566.

[32] Ian Li, Anind K Dey, and Jodi Forlizzi. 2011. Understanding my data, myself: supporting self-reflection with ubicomp technologies. In Proceedings of the 13th international conference on Ubiquitous computing. ACM, 405-414.

[33] Edwin A Locke and Gary P Latham. 2002. Building a practically useful theory of goal setting and task motivation: A 35-year odyssey. American psychologist 57, 9 (2002), 705. 
[34] Edward McAuley and Bryan Blissmer. 2000. Self-efficacy determinants and consequences of physical activity. Exerc Sport Sci Rev 28, 2 (2000), 85-88.

[35] Andreas Möller, Matthias Kranz, Barbara Schmid, Luis Roalter, and Stefan Diewald. 2013. Investigating Self-Reporting Behavior in Long-Term Studies. In Proceedings of the SIGCHI Conference on Human Factors in Computing Systems (Paris, France) (CHI '13). Association for Computing Machinery, New York, NY, USA, 2931-2940. https://doi.org/10.1145/2470654.2481406

[36] Sean A Munson and Sunny Consolvo. 2012. Exploring goal-setting, rewards, self-monitoring, and sharing to motivate physical activity. In 2012 6th international conference on pervasive computing technologies for healthcare (pervasivehealth) and workshops. IEEE, 25-32.

[37] Jasmin Niess and Paweł W Woźniak. 2018. Supporting meaningful personal fitness: The tracker goal evolution model. In Proceedings of the 2018 CHI Conference on Human Factors in Computing Systems. 1-12.

[38] Faryle Nothwehr and Jingzhen Yang. 2007. Goal setting frequency and the use of behavioral strategies related to diet and physical activity. Health education research 22, 4 (2007), 532-538.

[39] António L Palmeira, Pedro J Teixeira, Teresa L Branco, Sandra S Martins, Cláudia S Minderico, José T Barata, Sidónio O Serpa, and Luís B Sardinha. 2007. Predicting short-term weight loss using four leading health behavior change theories. International fournal of Behavioral Nutrition and Physical Activity 4, 1 (2007), 14.

[40] Charlie Pinder, Jo Vermeulen, Benjamin R Cowan, and Russell Beale. 2018. Digital behaviour change interventions to break and form habits. ACM Transactions on Computer-Human Interaction (TOCHI) 25, 3 (2018), 15.

[41] Anne A Poulsen and Jenny M Ziviani. 2004. Can I play too? Physical activity engagement of children with developmental coordination disorders. Canadian fournal of Occupational Therapy 71, 2 (2004), 100-107.

[42] Mashfiqui Rabbi, Min Hane Aung, Mi Zhang, and Tanzeem Choudhury. 2015. MyBehavior: automatic personalized health feedback from user behaviors and preferences using smartphones. In Proceedings of the 2015 ACM International foint Conference on Pervasive and Ubiquitous Computing. ACM, 707-718.

[43] Chris J Riddoch, Calum Mattocks, Kevin Deere, Jo Saunders, Jo Kirkby, Kate Tilling, Sam D Leary, Steven N Blair, and Andy R Ness. 2007. Objective measurement of levels and patterns of physical activity. Archives of disease in childhood 92, 11 (2007), 963-969.

[44] William T Riley, Daniel E Rivera, Audie A Atienza, Wendy Nilsen, Susannah M Allison, and Robin Mermelstein. 2011. Health behavior models in the age of mobile interventions: are our theories up to the task? Translational behavioral medicine 1, 1 (2011), 53-71.

[45] John Rooksby, Mattias Rost, Alistair Morrison, and Matthew Chalmers. 2014. Personal tracking as lived informatics. In Proceedings of the SIGCHI conference on human factors in computing systems. ACM, 1163-1172.

[46] Jo Salmon, Neville Owen, David Crawford, Adrian Bauman, and James F Sallis. 2003. Physical activity and sedentary behavior: a population-based study of barriers, enjoyment, and preference. Health psychology 22, 2 (2003), 178.

[47] Dale H Schunk. 1990. Goal setting and self-efficacy during self-regulated learning. Educational psychologist 25, 1 (1990), 71-86.

[48] Grace Shin, Yuanyuan Feng, Mohammad Hossein Jarrahi, and Nicci Gafinowitz. 2019. Beyond novelty effect: a mixed-methods exploration into the motivation for long-term activity tracker use. JAMIA open 2, 1 (2019), 62-72.

[49] Mimmi Sjöklint, Ioanna D Constantiou, and Matthias Trier. 2015. The complexities of self-tracking-An inquiry into user reactions and goal attainment. Available at SSRN 2611193 (2015).

[50] Alexander D Stajkovic and Fred Luthans. 2003. Social cognitive theory and self-efficacy: Implications for motivation theory and practice. Motivation and work behavior 126 (2003), 140.

[51] Simon Stusak, Aurélien Tabard, Franziska Sauka, Rohit Ashok Khot, and Andreas Butz. 2014. Activity sculptures: Exploring the impact of physical visualizations on running activity. IEEE Transactions on Visualization and Computer Graphics 20, 12 (2014), 2201-2210.

[52] Lie Ming Tang and Judy Kay. 2017. Harnessing Long Term Physical Activity Data-How Long-term Trackers Use Data and How an Adherence-based Interface Supports New Insights. Proceedings of the ACM on Interactive, Mobile, Wearable and Ubiquitous Technologies 1,2 (2017), 26.

[53] Catrine E Tudor-Locke and Anita M Myers. 2001. Challenges and opportunities for measuring physical activity in sedentary adults. Sports medicine 31, 2 (2001), 91-100.

[54] William H Turnley and Daniel C Feldman. 2000. Re-examining the effects of psychological contract violations: unmet expectations and job dissatisfaction as mediators. fournal of organizational behavior 21, 1 (2000), 25-42.

[55] Amos Tversky and Daniel Kahneman. 1974. Judgment under uncertainty: Heuristics and biases. science 185, 4157 (1974), $1124-1131$.

[56] Luc Vanhees, Johan Lefevre, Renaat Philippaerts, Martine Martens, Wim Huygens, Thierry Troosters, and Gaston Beunen. 2005. How to assess physical activity? How to assess physical fitness? European fournal of Cardiovascular Prevention \& Rehabilitation 12, 2 (2005), $102-114$

Proc. ACM Interact. Mob. Wearable Ubiquitous Technol., Vol. 4, No. 4, Article 0. Publication date: 2020. 